\title{
Les arts visuels et la promotion de la santé du sein: Points de vue d'artistes
}

\author{
Par Barbara Thomas, Renée S. Marshall, \\ Susan B. Gold/Smith et Anne Forrest
}

\begin{abstract}
Note du traducteur
Pour alléger le texte, nous avons utilisé le féminin dans cet article pour désigner l'ensemble des artistes, bien que celui-ci comprenait quelques hommes.
\end{abstract}

\section{Résumé}

Nous devons disposer de méthodes spécialement conçues si nous souhaitons répondre aux besoins de certaines populations en matière de promotion de la santé. Cette étude présente une approche pluridisciplinaire née des efforts coopératifs de personnes ouvrant dans les domaines des sciences infirmières, des arts visuels et des études de la femme, le but de cette approche étant de promouvoir la santé du sein au moyen des arts visuels. L'objectif de cet article est de décrire le projet du point de vue des artistes, d'approfondir la compréhension de la santé du sein et de comprendre l'utilité des arts visuels en tant qu'outils de promotion de la santé. Nous avons adopté un protocole d'entrevue structurée et avons soumis les données d'entrevue à une analyse thématique. Les trois principaux thèmes qui se sont dégagés étaient les suivants: 1) un lien personnel fort avec le cancer du sein et la perception de la menace du cancer; 2) le besoin et le désir de promouvoir la santé au sein de la collectivité; 3) la nature unidimensionnelle du cancer du sein et de la santé du sein. Les entrevues ont révélé que les arts visuels constituent une méthode de promotion de la santé du sein à la fois novatrice et adaptative.

La promotion de la santé est un processus qui permet aux individus d'examiner et de comprendre leur propre vie en tant que reflet d'un idéal (Koithan, 1994). De nombreuses personnes ont avancé que la promotion de la santé passe par la convergence de modes multiples de sensibilisation et de compréhension personnelles (p. ex. Breslin, 1996; Carper, 1978; Koithan, 1994, 1996). Traditionnellement, la promotion de la santé a eu recours à la recherche empirique. Toutefois, il nous faut des approches personnalisées si nous souhaitons atteindre diverses populations. Les scientifiques, y compris les infirmières, n'ont pas toujours desservi certaines populations de façon efficace, par exemple les femmes, les enfants et les minorités (Vezeau, 1994). Les méthodes traditionnelles de promotion de la santé à l'intention du grand public ont souvent eu recours à des documents écrits pour des personnes bien instruites. Toutefois, dans le cas de personnes moins éduquées, de personnes illettrées ou de personnes qui ne maîtrisent pas la langue officielle, ces méthodes constituent souvent des entraves à l'éducation sanitaire. Les personnes de statut socio-économique moins élevé n'ont souvent pas accès aux initiatives de promotion de la santé. Ainsi, pour atteindre ces populations, il convient de considérer de nouvelles méthodes pédagogiques et, en dernier ressort, de nouvelles méthodes de promotion de la santé. L'esthétique constitue une façon de présenter l'expérience et les émotions personnelles qui ne peuvent se quantifier (Koithan, 1994). Cette méthode peut s'avérer efficace auprès des individus qui sont mal servis par les approches pédagogiques traditionnelles.

L'esthétique est une façon d'appréhender et de comprendre la signification de la vie. Il s'agit d'une approche créative permettant aux individus de concevoir et d'exprimer des possibilités à autrui et de découvrir de nouvelles connaissances (Cumbie et Rutherfoord,
1994). Les méthodes qui intègrent l'esthétique sont uniques en leur genre puisque ces modes de conscientisation agissent au niveau de la signification et sont capables de promouvoir la santé sur le plan de l'idéal et du soi (Koithan, 1994). Ainsi, les personnes peuvent saisir les concepts liés à la santé et les appliquer à leur propre vie. Les connaissances véhiculées par l'esthétique sont également importantes pour la compréhension de concepts qui ne peuvent se décrire ou s'expliquer facilement (Carper, 1978; Koithan, 1994). Cette approche peut atteindre des populations qui sont peut-être insuffisamment encadrées par les approches traditionnelles aux soins de santé, telles que les femmes de différents âges et les groupes ethniques.

Les sciences humaines, y compris les arts visuels, englobent la compréhension du sens de la vie humaine tant sur le plan individuel que sur le plan collectif (Bruderle et Valiga, 1994). L'art constitue une forme d'expression et de communication qui facilite l'échange de messages. À travers l'art, il est possible d'acquérir l'habileté de saisir et de vivre les sentiments et les expériences d'autrui (Hoshiko, 1985; Mohr, 1995). Si l'on donne à un observateur, par le biais de l'art, l'occasion de réfléchir à certaines questions précises liées à la santé, cette personne sera en mesure de promouvoir sa propre santé de même que celle d'autrui (Mitchell et Cody, 2002). Tout comme l'esthétique, l'art véhicule des messages au sujet d'expériences de vie et de santé et les met en lumière; il possède le potentiel d'influencer les pratiques sanitaires des individus. Dans le cadre de ce projet novateur sur la santé du sein, les principes de l'esthétique et de l'art ont été combinés, en particulier leur capacité de communiquer de façon non traditionnelle. Ce projet destiné aux femmes a été financé par une fondation nationale du cancer du sein.

L'objectif du projet était de sensibiliser les femmes à la santé du sein et de promouvoir la santé du sein par la création d'œuvres d'art visuelles et par la présentation de ces œuvres à une vaste gamme de femmes. Le projet a adopté une approche pluridisciplinaire regroupant des personnes œuvrant dans les domaines des sciences infirmières, des arts visuels et des études de la femme. Cette approche a été choisie en raison du fait que la promotion de la santé n'appartient à aucun de ces trois domaines en particulier ni aux seules professions de la santé. Dans le cadre du projet, les sciences infirmières ont couvert le volet promotion de la santé, tandis que les arts visuels ont ciblé la production artistique thématique et que les études de la femme s'intéressaient aux avantages de la promotion de la femme et de sa santé. Des professeures universitaires, des étudiantes et des membres de la collectivité ont créé des œuvres d'art qui ont ensuite été exposées dans la collectivité. Certaines œuvres ont également été affichées dans le site Web du projet affilié à l'université, afin d'augmenter le nombre de femmes touchées par cette campagne de promotion de la santé du sein (Thomas,

Barbara Thomas, RN, EdD, est professeur émérite à la Faculté des sciences infirmières, Université de Windsor, Windsor, Ontario. Renée S. Marshall, B.Sc. (Biochimie), RN, BSc.N., Université de Windsor. Susan B. Gold/Smith, MA, est professeure, École des arts visuels, Université de Windsor, Windsor, Ontario. Anne Forrest, PhD, est Professeure agrégée, Faculté d'administration des affaires et directrice, Women's Studies Program, à l'Université de Windsor, Windsor, Ontario. 
Gold/Smith, Forrest et Marshall, 2002). Ce site Web est disponible à www.uwindsor.ca/eob. Les artistes qui ont participé au projet ont ensuite été interviewées afin de connaître leurs points de vue relativement à la santé du sein et de mieux comprendre comment les arts visuels peuvent servir à promouvoir la santé. Cet article présente et analyse les données d'entrevue. Les autres volets du projet dépassent la portée de cet article.

\section{Méthodologie}

\section{Participants}

Au total, 37 artistes ont pris part au projet sur la santé du sein. La plupart des participants étaient des femmes $(86 \%, \mathrm{n}=32)$. Parmi ces 32 femmes, trois avaient survécu au cancer $(8 \%)$. Bien que l'on ait approché tous les artistes pour une entrevue, seules 24 personnes y ont participé. Quatre des artistes se sont montrées intéressées à faire une entrevue mais n'ont pu la réaliser en raison de conflits d'horaires, tandis que neuf autres personnes n'ont pu être jointes. La majorité des personnes interviewées étaient des femmes $(83 \%, \mathrm{n}=20)$, dont deux des trois femmes qui avaient survécu au cancer. Les artistes et les personnes interviewées appartenaient à trois groupes: étudiantes, professeures d'université et membres de la collectivité (voir la figure 1). Les données d'entrevue ont été recueillies au cours d'une période de huit mois.

\section{Démarche}

On a initialement contacté les artistes par téléphone et par courrier électronique afin de les inviter à prendre part à une entrevue. On a tenté de communiquer avec chaque artiste quatre fois. L'intervieweuse et l'artiste ont convenu de se rencontrer à un moment convenable pour les deux. L'intervieweuse a commencé l'entrevue en obtenant le consentement éclairé de l'artiste, et chaque personne interviewée a reçu une copie d'une lettre d'information et du formulaire de consentement. On a adopté un protocole d'entrevue structurée afin d'assurer la cohérence entre les entrevues. On a obtenu l'approbation du comité universitaire de déontologie pour la recherche impliquant des sujets humains. Les chercheuses ont collaboré à l'élaboration d'un guide d'entrevue comprenant dix questions ouvertes basées sur leur expérience et sur les publications recensées. Ces questions étaient conçues pour faciliter la discussion au sujet de la participation à un projet sur la santé du sein. L'intervieweuse notait les réponses des participantes sous forme schématique. Entre les questions, l'intervieweuse relisait la réponse à la question précédente pour en assurer l'exactitude et pour donner l'occasion à l'artiste d'ajouter des commentaires. Les notes de l'intervieweuse étaient transcrites

Figure 1. Nombre d'artistes participants au projet et nombre d'entre eux ayant participé à l'entrevue, par groupes.

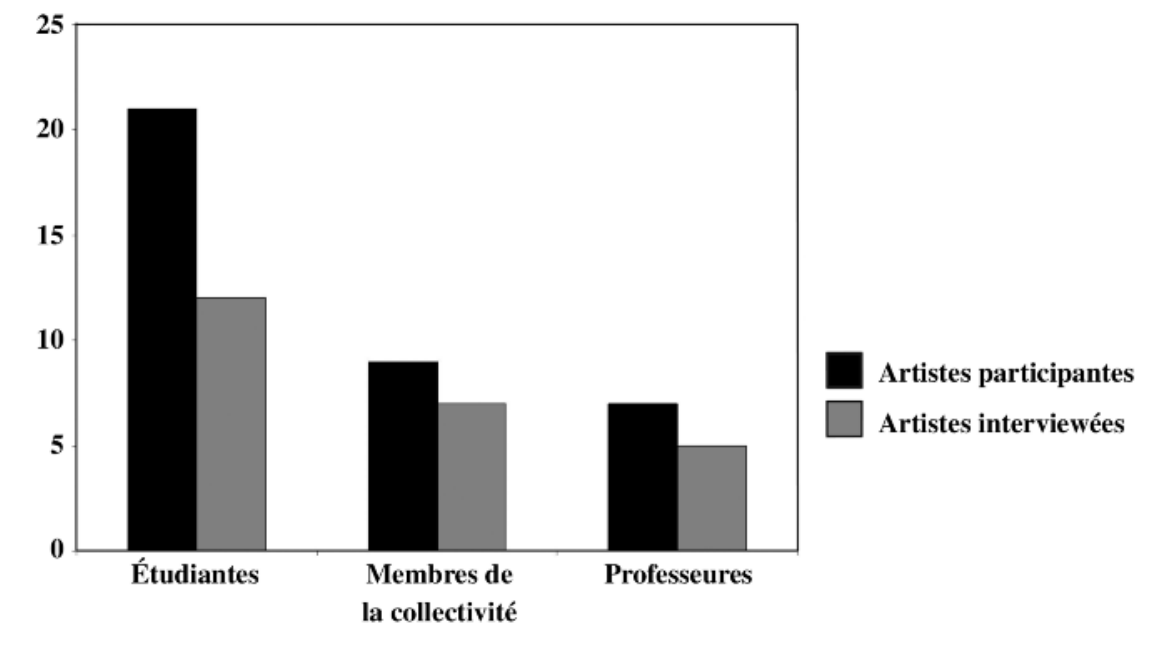

immédiatement après l'entrevue afin de maintenir l'intégrité des données et d'assurer l'exactitude du cadre de référence. Pour des raisons de cohérence, les données ont été recueillies et analysées par une seule intervieweuse. Les chercheuses ont surveillé l'analyse des données afin d'assurer la fiabilité et la validité du processus. L'intervieweuse connaissait le projet puisqu'elle y participait depuis le début. Grâce à l'utilisation du format d'entrevue structurée et à l'excellent niveau de connaissances de l'intervieweuse au sujet du projet et du processus d'entrevue, cette dernière a pu réaliser la collecte et l'analyse des données (Polit et Beck, 2003). Seules les chercheuses avaient accès aux données d'entrevue; à des fins de confidentialité, on a utilisé des pseudonymes dans cet article. Les notes de terrain, les formulaires de consentement signés et les entrevues dactylographiées ont été entreposés de façon sécuritaire.

On a utilisé trois modèles d'entrevue: en personne dans la maison de l'artiste, en personne sur le campus de l'université ou par téléphone. Dans chaque cas, on a tenu compte des préférences de l'artiste lors du choix de l'endroit où aurait lieu l'entrevue. Quinze des artistes ont préféré une entrevue en personne tandis que les neuf autres ont choisi l'option téléphonique.

\section{Analyse des données}

Une fois toutes les entrevues terminées, le contenu a été analysé des points de vue thématique et conceptuel. Une analyse thématique à la main a également été réalisée. L'examen des données a mené à l'élaboration d'une classification thématique codifiée basée sur la fréquence des réponses et sur la relation au sujet examiné. Ceci a permis de quantifier les réponses en fonction des thèmes. Les classifications n'étaient pas exhaustives, puisque certaines données n'étaient pas pertinentes pour l'enquête globale. Sept des dix questions ont reçu un code thématique tandis que les trois autres n'en ont pas reçu. Ainsi, les deux dernières questions de l'entrevue n'ont pas été quantifiées puisqu'un seul thème s'en est dégagé, rendant ainsi la codification non nécessaire. La troisième question non codifiée portait sur le processus créatif et n'a pas été classifiée en raison de la divergence importante au sein des réponses.

\section{Résultats}

On a examiné les thèmes qui se sont dégagés des entrevues en fonction des questions correspondant aux réponses fournies. Les deux dernières questions de l'entrevue figurent ensemble puisqu'elles sont reliées et appartiennent au même thème. Les questions étaient ouvertes, si bien que les réponses à la même question appartenaient souvent à plus d'un thème.

Question: Qu'est-ce qui vous a initialement incitée à prendre part à ce projet?

Les trois principaux thèmes qui se sont dégagés des réponses à cette question étaient les suivants: 1) un lien personnel avec l'université; 2) un lien personnel avec le cancer du sein; 3) la nature inspirante du projet. Dix-sept $(71 \%)$ des artistes interviewées ont initialement été attirées au projet de par leur lien personnel avec l'université. Certaines étudiaient à l'université, d'autres y enseignaient, connaissaient les chercheuses ou avaient vu les annonces affichées sur le campus. Quarante-deux pour cent $(n=10)$ des artistes ont été attirées par le projet en raison d'un lien personnel avec le cancer du sein. Certaines avaient survécu elles-mêmes au cancer du sein, tandis que d'autres avaient des proches ou des amies touchées par cette maladie. Certaines artistes avaient également un lien avec la communauté du cancer du sein. Parmi ces dix participantes, plusieurs ont indiqué se sentir personnellement menacées par le cancer du sein. Pour certaines de ces artistes, la participation au 
projet constituait une façon d'exprimer leurs propres expériences en matière de santé du sein. Une des artistes s'est dite attirée par le changement que pourra éventuellement opérer le projet dans le domaine de la santé du sein et a exprimé son point de vue personnel, né de son expérience avec cette maladie du sein. D'autres artistes ont affirmé avoir été attirées par le projet en raison de l'expérience d'amies ou de membres de leur famille en matière de santé du sein. Par exemple, une des artistes a senti le besoin de participer après avoir vu une amie combattre le cancer du sein et recouvrer la santé par la suite. Neuf des artistes interviewées (38\%) étaient d'avis que le projet était à la fois intéressant et important. Elles voyaient le projet comme un outil de sensibilisation capable de favoriser le changement et de promouvoir la santé parmi les femmes. Elles ont donc décidé de s'impliquer. Une des artistes a décrit sa peur du cancer du sein et a affirmé que "l'éducation en matière de santé du sein est insuffisante" (Jane), et que le projet représentait "une cause juste" (Jane). De nombreuses artistes interviewées ont également exprimé que cette même peur les a motivées à participer, dans l'espoir de promouvoir la santé parmi les femmes.

Question: Comment avez-vous commencé à réfléchir au thème de l'exposition (santé du sein)?

Les personnes interviewées ont adopté trois grandes perspectives dans leur approche à la santé du sein. Cinquante-quatre pour cent $(\mathrm{n}=$ 13) d'entre elles ont adopté une perspective médicale axée sur le cancer du sein. Par exemple, certaines ont centré leur travail sur le risque, en pourcentage, de contracter le cancer du sein au cours de la vie. Une des artistes s'est concentrée sur l'aspect pathologique du thème en raison de son expérience personnelle avec le cancer du sein. Comme elle l'a expliqué, "mon travail est né de ma colère, de ma volonté de lutter, de guérir et d'y survivre" (Sally). Seulement huit des artistes interviewées (33\%) ont traité le thème du projet comme étant la santé du sein. Leur travail soulignait des méthodes de promotion de la santé du sein et d'éducation parmi les femmes et les hommes. Sept (29\%) des personnes interrogées ont adopté une perspective axée sur les divers aspects du sein, y compris son esthétique, sa fonction pour les hommes et pour les femmes, sa signification et enfin, son rôle fascinant dans la grossesse. Une des artistes a décrit son approche au thème de la santé du sein comme étant une vision idéale du sein. Ayant décrit le cancer comme un objet invisible, elle a axé son travail sur l'aspect esthétique du sein.

Question: Quelles étaient vos sources d'information pour le projet?

La plupart des personnes interviewées ont indiqué que leurs principales sources d'information étaient la recherche et la discussion $(71 \%, \mathrm{n}=17)$. Parmi les ressources de recherche, on comptait les livres, la documentation médicale, les ressources Internet, les vidéos et les présentations. Au cours de leur travail, les artistes ont eu de nombreuses discussions avec des personnes qui facilitaient le processus créatif, telles que des membres de leur famille, des amis, des collègues artistes et d'autres femmes. Onze des artistes interviewées $(46 \%)$ ont cité d'autres sources d'information telles l'expérience personnelle, les connaissances personnelles et les expériences de travail. L'expérience personnelle comprenait des circonstances de leur propre vie ou celle d'amis ou de proches; ces expériences avaient marqué leur vie et subséquemment touché leur travail artistique. Les connaissances personnelles désignaient des idées et des concepts liés à la santé et aux seins, des idées que l'artiste possédait déjà avant le début du projet. Ces idées et concepts n'étaient donc pas nés de la recherche liée au projet.

Question: Quels facteurs ont influencé votre travail (c.-à-d. la collaboration entre les disciplines des arts visuels, des sciences infirmières et des études de la femme)?

Plusieurs des sources d'information se sont également révélées être des facteurs d'influence pour les artistes interviewées. Les expériences personnelles ont influencé $79 \%(\mathrm{n}=19)$ des artistes interrogées. Ces expériences comprenaient l'observation d'amies ou de membres de la famille aux prises avec le cancer du sein, des expériences personnelles avec la santé du sein et leur propre travail artistique passé. Les idées et expériences d'amies et de membres de la

famille au sujet du cancer du sein ont influencé 11 (46\%) des artistes interviewées. Les résultats de la recherche ont également influencé les artistes et leur travail dans $29 \%$ des cas $(n=7)$. Ceci comprenait toute connaissance acquise au cours du projet ayant eu une incidence sur l'orientation, la conception ou la réalisation du travail artistique. Les artistes interrogées ont indiqué que les livres, tels que ceux portant sur la médecine, sur des questions féminines et sur l'art, avaient influencé leur travail. Elles ont également mentionné d'autres sources de renseignements telles Internet, les vidéos et les documentaires.

Question: Comment avez-vous lié votre travail en studio au thème du projet?

Les éléments symboliques entourant la femme, le corps féminin et la santé du sein constituaient le principal lien entre le travail artistique et le thème du projet. Soixante-quinze pour cent $(n=18)$ des artistes interviewées ont indiqué que leur symbolisme s'était manifesté par l'utilisation d'images $(n=6)$ et de couleurs $(n=4)$, ou par la présentation $(n=5)$ et les matériaux employés $(n=12)$. Certaines des artistes interrogées ont lié leur travail au thème par le biais des images qu'elles ont choisi de dépeindre. Par exemple, l'une d'entre elles a choisi une image déformée, symbolisant ainsi la distorsion causée par la maladie au sein de la famille. Pour d'autres, l'emploi de couleurs constituait le lien entre le thème et la création artistique. Par exemple, une des artistes a utilisé le rose pour symboliser le ruban rose associé au cancer du sein. D'autres artistes ont indiqué que leur lien avec le thème s'était manifesté dans la présentation de leur travail, p. ex. dans le choix d'un cadre doré. Pour cette artiste, l'or constituait une ressource précieuse; elle a donc choisi un cadre doré pour symboliser la grande valeur des seins. Certaines artistes ont utilisé différents matériaux pour symboliser le lien entre leur travail et la santé du sein. Par exemple, une des artistes interviewées a choisi un matériau très fragile pour symboliser "la fragilité de la femme et son incapacité de se défendre contre cette maladie" (Mary).

La représentation de la femme dans les médias et les pressions exercées sur la femme pour qu'elle se conforme à des idéaux sociaux constituaient, en ordre d'importance, le second lien entre le travail artistique et le thème du projet. Sept des artistes interrogées $(29 \%)$ se sont dites préoccupées par la représentation de la femme dans les médias et par les pressions qui pèsent sur la femme pour qu'elle se plie à un idéal social. Ces artistes ont utilisé leur travail pour dépeindre la santé du sein non pas en tant que norme ou idéal mais plutôt en tant que véhicule permettant à la femme de se connaître ellemême. Pour citer une des artistes, "Les femmes transforment beaucoup leur corps, par exemple par la chirurgie, pour ressembler à

\section{Tableau 1: Facteurs imprévus ayant un effet sur les œuvres d'art}

\begin{tabular}{|l|c|}
\hline Facteur & $\begin{array}{c}\text { Nombre de } \\
\text { répondants }\end{array}$ \\
\hline Difficultés avec les matériaux, les images & 7 \\
Rétroaction d'autrui & 4 \\
Résultat final inattendu/surprenant & 4 \\
Aucun & 2 \\
Contraintes temporelles du projet & 2 \\
Amélioration de la sensibilisation/ & \\
de l'appréciation de l'œuvre et de la & \\
relation à la santé des femmes & 2 \\
Questions d'ordre personnel/ & 1 \\
liées à la santé personnelle & 1 \\
Expérience de guérison & 1 \\
Collaboration/liens d'amitié avec co-artiste & 1 \\
Transport de l'œuvre & \\
\hline
\end{tabular}


l'idéal social. Les seins font partie intégrante du corps, donc pourquoi ne pas être qui nous sommes? Il n'y a aucune raison de suivre une norme fixe" (Sue).

Question: Vos idées concernant le thème ont-elles évolué au cours de votre travail?

Quelques artistes interrogées $(38 \%, \mathrm{n}=9)$ ont indiqué que la nature de leur travail artistique avait évolué au cours du processus, que ce soit du point de vue de la conception, des dimensions, du lien avec le thème ou du processus créatif lui-même. Ces changements, auxquels a contribué l'information découlant de la recherche, ont influencé les images utilisées, la taille des œuvres et les matériaux employés. Les commentaires d'autrui et les contraintes temporelles ont également entraîné des changements sur le plan artistique. Vingtcinq pour cent $(n=6)$ des artistes interviewées n'ont pas modifié leurs idées au cours du projet.

Question: Votre travail a-t-il été influencé par d'autres facteurs, qu'ils aient été liés au projet ou non, prévus ou non?

Les taux de réponse concernant les facteurs imprévus qui ont eu une incidence sur les artistes et leur travail figurent au tableau 1. Dans $29 \%(\mathrm{n}=7)$ des cas où des facteurs imprévus avaient eu un effet sur le travail, ceux-ci étaient dus à des difficultés avec les matériaux et avec les images produites. Les deux autres facteurs principaux ayant modifié le travail des artistes étaient la rétroaction d'autrui et les résultats finaux surprenants ou inattendus $(17 \% ; n=4$ dans chaque cas). Les collègues artistes, les membres de la famille et les amis étaient les principales sources d'influence sur le cours des idées des artistes et sur leurs œuvres subséquentes. Certaines artistes interrogées ont indiqué que leur travail final était différent de ce qu'elles avaient prévu. Par exemple, Linda a décrit le processus artistique comme "une évolution" de ses pensées, sentiments et idées par rapport à la santé du sein.

Question: Qu'est-ce que la participation à ce projet a signifié pour vous? Avez-vous d'autres commentaires au sujet de la participation à ce projet coopératif axé sur la santé du sein?

Toutes les artistes interviewées ont exprimé des commentaires positifs lorsqu'on leur a demandé ce qu'avait signifié pour elles la participation au projet. Plusieurs se sont dites fières de prêter leur appui et de prendre part à une initiative communautaire de promotion de la santé des femmes, et étaient heureuses de participer à un projet interdisciplinaire. Pour citer une des artistes, "C'était très gratifiant, tant sur le plan personnel que professionnel, de travailler avec des personnes venant d'autres disciplines. J'ai grandement apprécié le fait que toutes les œuvres d'art étaient à la fois pertinentes et expressives pour l'ensemble d'une vaste communauté" (Linda). Une autre artiste a résumé ses pensées concernant le projet en affirmant que ce dernier constituait une "contribution positive à la vie" (Sheila), tandis qu'une troisième artiste était fière "de prêter son appui et de participer à une initiative communautaire visant à promouvoir la santé des femmes" (Kathy). Les artistes interviewées ont indiqué que le projet avait contribué à la promotion de la santé parmi les artistes elles-mêmes tandis qu'elles se renseignaient et examinaient leur propre santé et celle d'autres personnes dans la collectivité. Une des artistes a illustré cette idée en affirmant:

Le projet m'a laissée sur ma faim. Je veux transformer le système de santé canadien dans son ensemble, embaucher plus de médecins, d'infirmières et de professionnels de la santé. La collaboration requise pour opérer une telle transformation doit nécessairement inclure des femmes et des hommes issus de nombreuses disciplines, des gouvernements et des médias (Anne).

\section{Discussion}

Dans presque tous les cas, les données d'entrevue ont révélé un lien profond entre le cancer du sein et les artistes interviewées. Ces dernières évoquaient un lien personnel avec le cancer du sein. Plusieurs y avaient elles-mêmes survécu, d'autres avaient des êtres chers qui en avaient souffert, tandis que d'autres encore se sentaient menacées par ce fléau. Pour citer l'une d'elles, “Toutes les femmes ont peur de développer un cancer du sein" (Jane). La recherche corrobore cette affirmation et indique que la sensibilisation du public a entraîné une augmentation de la peur, en particulier parmi les femmes dont la famille a été touchée par le cancer du sein. De plus, le fait d'être proche d'un membre de la famille atteint du cancer du sein influence les pratiques des femmes en matière de santé du sein (Chalmers et Luker, 1996). On estime que les femmes en Ontario ont une chance sur neuf de développer un cancer du sein au cours de leur vie (Cancer Care Ontario, 2002); il est donc probable que tous les gens soient touchés, directement ou indirectement, par le cancer du sein. Les réponses obtenues dans le cadre des entrevues reflétaient ces résultats, puisqu'aucune des artistes ne se sentait à l'abri de cette maladie.

Les réponses aux questions d'entrevue ont presque toutes indiqué la perception de la menace du cancer du sein. Un des aspects intéressants de ces réponses concerne l'âge des personnes interviewées, puisque la moitié d'entre elles étaient des étudiantes de moins de 40 ans. Dans certains cas, la recherche indique que la peur du cancer peut empêcher une jeune femme de se mettre en quête de soins de santé (Howell, Huelsman, Everett et Hopkin, 2002). Une des tâches des jeunes adultes est de se former une identité personnelle. Il est facile d'acquérir tôt dans la vie de bonnes habitudes de santé de façon à ce qu'elles fassent partie du quotidien et de l'identité personnelle; ces habitudes peuvent avoir des effets positifs tout au long de la vie (Ludwick et Gaczkowski, 2001; Maurer, 1997). Cette étude indique que les jeunes adultes sont à un âge où l'éducation et la promotion de styles de vie sains sont importants.

Tel que l'avaient anticipé les chercheuses, la plupart des artistes interviewées se sentaient attirées par le projet en raison d'un lien personnel avec l'université. Il se peut que l'ambiance universitaire ait contribué au taux de participation des artistes, puisqu'il est notoire que l'université constitue un milieu très favorable à la présentation et à l'étude de nouvelles idées et de nouvelles options (Maurer, 1997). De nombreuses artistes ont également été attirées par le projet en raison de l'importance de la santé du sein et d'un intérêt pour la sensibilisation par rapport à la santé et pour la promotion de cette dernière. Les artistes souhaitaient que leur participation "opère un changement" (Anne) et y voyaient "une occasion de contribuer à un enjeu important" (Linda). Howell et coll. (2002) ont recensé des attitudes semblables par rapport au cancer du sein. Ils ont signalé l'importance de diffuser plus de renseignements au sujet du cancer et de sensibiliser la population.

Les partenariats entre la collectivité et l'université sont en mesure de promouvoir la santé des collectivités (Meade et Calvo, 2001). La recherche a démontré que ces dernières sont davantage disposées à participer à des partenariats d'apprentissage si elles y voient un avantage pour elles (Oneha, Magnussen et Feletti, 1998). Les résultats de cette étude indiquent que les artistes interviewées, qui sont des membres de la collectivité, estimaient que le projet avait la capacité de promouvoir la santé, et elles le percevaient comme bénéfique. Les artistes ont montré qu'elles valorisaient la promotion de la santé du sein par leur soif d'information et leur besoin de discuter de ces enjeux avec leurs amis, leurs proches et d'autres membres de la collectivité. Les réponses indiquaient que les membres de la collectivité souhaitent jouer un rôle actif dans leur propre santé et dans celle de leur collectivité. Ceci illustre la nécessité de lancer davantage d'initiatives communautaires visant à promouvoir la santé pour l'ensemble de la population, en particulier des partenariats entre universités et collectivités.

Bien que le projet était axé sur la santé du sein, plus de la moitié des personnes interviewées ont adopté une approche centrée sur le thème du cancer du sein et sur l'aspect médical du thème. Cette interprétation était directe et démontrait un désir de la part des artistes de sensibiliser la population à la réalité du cancer et à la menace qu'il représente pour toutes les femmes. Étant donné que plusieurs des artistes interviewées 
étaient influencées par un lien personnel avec le cancer du sein, elles se sont concentrées davantage sur la maladie que sur la santé du sein. Il est intéressant de noter que de nombreuses études ont indiqué que les femmes attribuent des significations positives à leurs expériences avec le cancer du sein (Degner, Hack, O’Neil et Kristjanson, 2003; Luker, Beaver, Leinster et Owens, 1996). Cependant, les femmes plus âgées ont indiqué qu'il leur aurait été plus difficile de voir l'aspect positif du cancer du sein si elles avaient été plus jeunes, et les femmes plus jeunes attribuaient effectivement des significations plus négatives à leur expérience avec le cancer (Degner et coll.). Le fait que tant d'artistes aient axé leur travail sur le cancer du sein souligne la nature unidimensionnelle de la question santé du sein/cancer du sein, en particulier parmi les jeunes femmes. Il se peut que ceci ait des implications pour les éducateurs et les responsables de la promotion de la santé. Les résultats de cette étude indiquent que les efforts pédagogiques ne devraient peut-être pas être axés exclusivement sur la santé. Il se peut qu'une approche plus directe centrée sur la prévention du cancer du sein soit plus appropriée.

Les deux tiers des personnes interviewées ont eu recours au symbolisme pour lier leur travail au thème du projet. Elles ont utilisé des images, des couleurs, la présentation du travail et divers matériaux pour représenter la femme et la santé du sein. Les œuvres d'art créées dans le cadre du projet adoptaient des approches variées pour véhiculer leur message respectif. Les programmes pédagogiques ciblant les populations vulnérables telles que les personnes âgées, les personnes à faible revenu et les minorités doivent être à la fois novateurs et sensibles (Wood, Duffy, Morris et Carnes, 2002). Ils doivent tenir compte des croyances culturelles de même que des niveaux d'alphabétisation des membres de la collectivité (Meade et Calvo, 2001). La recherche a également démontré que la combinaison d'outils pédagogiques est plus efficace que l'utilisation d'outils individuels, et que les initiatives de promotion de la santé devraient être interactives, particulièrement pour les jeunes. De plus, les personnes s'identifient plus facilement aux outils qui dépeignent des personnes réelles et leurs expériences vécues, puisque ceci augmente la pertinence des outils et du message (Howell et coll., 2002). En tant qu'outils de promotion de la santé du sein, les arts visuels étaient idéaux pour les populations ciblées parce qu'ils tenaient compte des considérations soulignées par la recherche. Le projet a atteint de nombreuses femmes grâce à la diversité des œuvres d'art créées et à la variété des opinions exprimées dans les entrevues. Chaque œuvre d'art avait son attrait propre et, en tant qu'effort collectif, l'ensemble des œuvres réunies a réussi à véhiculer une multitude de messages et d'émotions au sujet de la santé du sein.

Les arts visuels constituent une stratégie novatrice de promotion de la santé. Afin d'améliorer cette approche, il convient d'allouer plus de temps à la création artistique, puisque les difficultés avec les matériaux et les influences d'autrui ont un effet considérable sur le travail. Avec plus de temps, les artistes auraient l'occasion de peaufiner leurs œuvres des points de vue de la créativité et de la clarté du message, ce qui aurait pour effet d'améliorer la capacité des œuvres de promouvoir la santé du sein dans la collectivité.

Pour résumer, les artistes qui ont pris part à cette étude souhaitent participer de façon plus active à la promotion de la santé du sein parmi les femmes de la collectivité. Elles étaient prêtes à partager avec autrui leurs nombreuses expériences personnelles avec le cancer et la santé du sein. La promotion de la santé du sein parmi les jeunes est appropriée et efficace si l'on souhaite encourager l'acquisition de bonnes pratiques de santé pour toute la vie, parce que les jeunes sont sensibles et réceptifs à l'information liée au cancer et à la santé du sein. Toutefois, la promotion de la santé du sein devrait comprendre la prévention du cancer du sein, puisque pour les femmes, il n'y a pas de différence entre la santé du sein et le cancer du sein. Les membres de la collectivité souhaitent que davantage de personnes s'impliquent dans la promotion de la santé au niveau communautaire. Les partenariats entre la collectivité et l'université peuvent offrir l'environnement idéal pour cette implication, en autant que les projets soient coopératifs et conçus pour la collectivité. L'utilisation des arts visuels en tant qu'outils de promotion de la santé constitue une nouveauté sensible aux enjeux culturels et circonstanciels et capable de diffuser des messages sur la santé du sein auprès d'un grand nombre de femmes et d'hommes.

\section{Limites}

Une des limites du projet est le nombre réduit d'artistes qui ont choisi de participer aux entrevues. En raison de l'affiliation du projet avec l'université, plusieurs de ces artistes étaient des étudiantes qui ont déménagé ou qui ont obtenu leur diplôme et n'ont pas laissé leur nouvelle adresse. De plus, plusieurs des artistes

\section{L'œuvre Keeping Abreast of Breast Cancer \\ Artistes: Louise Chance Baxter et lain Baxter Photographie en couleur}

L'œuvre Keeping Abreast of Breast Cancer est une photographie en couleur de grand format. En effet, elle mesure environ 2,5 pieds de large et 3 pieds de haut. Son encadrement professionnel se compose d'un cache noir et d'un simple cadre doré. Elle a pour sujet les seins d'une femme entre lesquels quatre livres ont été glissés. Le cadrage est tel que les bords latéraux du cache touchent presque le côté de chaque sein. On ne voit aucune autre partie du corps de la femme. Ses seins, de grande taille, reposent sur une table ou une surface plane. Cette dernière est recouverte d'un tissu à motif qui se compose de grosses fleurs roses et bleues. Il fait penser au tissu qui sert à faire des draps ou des nappes. La femme de la photo porte une robe de chambre jaune citron en tissu éponge qui n'apparaît que dans les coins supérieurs de la photographie. Les livres placés entre les deux seins se rapportent tous au cancer du sein. Ils ont pour titre "Caring for the Patient with Breast Cancer" (le livre rose), "Breast Cancer" (le livre noir et jaune) alors qu'il est impossible de discerner le titre du livre blanc. Le dernier livre, noir, a aussi pour titre "Breast Cancer". Les seins de la photographie servent de serre-livres symboliques aux livres renseignant sur le cancer du sein. La robe de chambre en tissu éponge et la série de livres sur les connaissances liées à la maladie et sur les soins à dispenser composent un véritable récit à propos d'une personne qui s'informe sur le cancer du sein, fait face à la maladie ou prend soin d'une personne qui en est atteinte.

Description réalisée par Ilene Sova

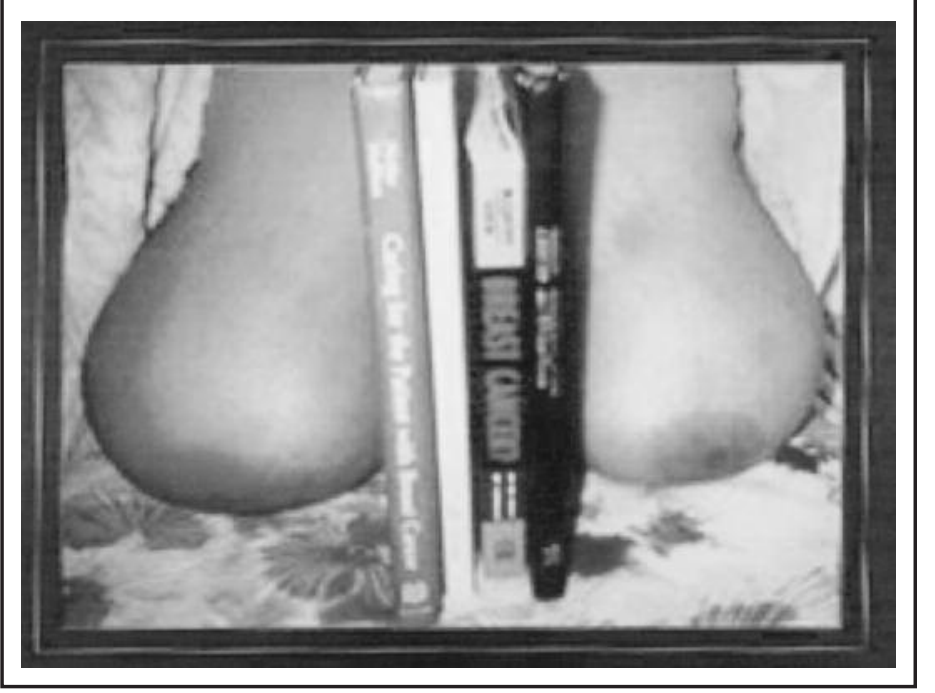


interviewées ne se sont pas présentées aux rendez-vous fixés, ce qui a réduit considérablement l'échantillon de personnes interviewées (voir le tableau 1). Les seules données démographiques recueillies auprès des artistes interviewées concernaient leur sexe, leurs antécédents de cancer du sein et leur affiliation avec le projet. Ceci a réduit l'éventail de résultats obtenus sur les sous-groupes de participants, tels que les idées caractéristiques de chaque groupe d'âge. En raison de la taille réduite de l'échantillon et de la nature délicate du sujet, une classification plus détaillée des participantes n'aurait pas permis de conserver l'anonymat de ces dernières et aurait diminué l'impact des résultats obtenus. Le manque de constance dans l' utilisation du questionnaire a constitué une autre limite du projet. Bien que les entrevues ont été menées par une seule et même personne et que l'on a utilisé un protocole d'entrevue structurée, il se peut que l'approche ait varié légèrement d'un jour à l'autre. L'utilisation d'un format d'entrevue plus structurée exigeant des réponses au moyen d'échelles, pourrait éventuellement améliorer les résultats et fournir des données quantitatives pour l'analyse statistique.

\section{Conclusion}

Ce projet et les entrevues subséquentes ont démontré la valeur des arts visuels dans la promotion de la santé du sein auprès de groupes importants de clients. Il s'agit d'une approche à la fois novatrice, adaptative et communicative. Les artistes souhaitent participer activement à la promotion de la santé et elles possèdent les aptitudes

\section{Références}

Breslin, E.T. (1996). Aesthetic methods as a means of knowing for nursing. Issues in Mental Health Nursing, 17(6), 503-505.

Bruderle, E.R., \& Valiga, T.M. (1994). Integrating the arts and humanities into nursing education. In P.L. Chinn \& J. Watson (Eds.), Art and aesthetics in nursing (pp. 117-144). New York: National League for Nursing Press.

Cancer Care Ontario. (2002). The Ontario breast screening program. Retrieved August 30, 2002, from http://www.cancercare.on.ca/obsp/english.htm

Carper, B.A. (1978). Fundamental patterns of knowing in nursing. Advances in Nursing Science, 1(1), 13-23.

Chalmers, K.I., \& Luker, K.A. (1996). Breast self-care practices in women with primary relatives with breast cancer. Journal of Advanced Nursing, 23(6), 1212-1220.

Cumbie, S.A., \& Rutherfoord, S.R. (1994). Weaving aesthetics into practice: The use of aesthetic techniques in group psychotherapy with clients remembering repressed traumatic memories. In P.L. Chinn \& J. Watson (Eds.), Art and aesthetics in nursing (pp. 223-245). New York: National League for Nursing Press.

Degner, L.F., Hack, T., O’Neil, J., \& Kristjanson, L.J. (2003). A new approach to eliciting meaning in the context of breast cancer. Cancer Nursing, 26(3), 169-178.

Hoshiko, B.R. (1985). Nursing diagnosis at the art museum. Nursing Outlook, 33(1), 32-36.

Howell, K.A., Huelsman, K.M., Everett, J.N., \& Hopkin, R.J. (2002). Breast cancer genetics education for college women: An evaluation of approaches. Journal of Cancer Education, 17(2), 74-77.

Koithan, M. (1994). The seeing self: Photography and storytelling as a health promotion methodology. In P.L. Chinn \& J. Watson (Eds.), Art and aesthetics in nursing (pp. 247-261). New York: National League for Nursing Press.

Koithan, M.S. (1996). Aesthetics in nursing practice and education. Issues in Mental Health Nursing, 17(6), 529-539.

Ludwick, R., Gaczkowski, T. (2001). Breast self-exams by teenagers: Outcome of a teaching program. Cancer Nursing, 24(4), 315-319. et connaissances requises pour contribuer à la santé des collectivités. Les approches pédagogiques à la santé du sein doivent adopter des techniques uniques en leur genre et tenir compte de la nature unidimensionnelle du cancer du sein et de la santé du sein. L'esthétique et l'art constituent des véhicules de connaissance et de définition de l'identité; à travers eux, nous pouvons nous connaître et proposer le changement.

Les arts puisent dans notre essence créatrice, cette partie de nous qui exprime la vie intérieure, l'imagination, la mémoire... Ils naissent de nos vies intérieures et constituent une oasis de répit et de fraîcheur dans un monde technologique et profondément stressant. De par leur capacité d'offrir ceci de façon particulière et significative, les arts visuels peuvent nous aider à nous comprendre nous-mêmes et à comprendre autrui de façon plus complète (Young-Mason, 2000, p. 197).

L'art constitue une stratégie viable de sensibilisation et de promotion de la santé.

\section{Remerciements}

Ce projet a été réalisé à l'Université de Windsor, à Windsor, en Ontario, au Canada. Il a reçu une subvention pour l'éducation communautaire de la part de la Fondation canadienne pour le cancer du sein, division de l'Ontario. Les chercheuses tiennent à remercier l'ensemble des artistes, en particulier celles et ceux qui ont partagé leurs pensées dans le cadre d'entrevues.
Luker, K.A., Beaver, K., Leinster, S.J., \& Owens, R.G. (1996). Meaning of illness for women with breast cancer. Journal of Advanced Nursing, 23(6), 1194-1201.

Maurer, F. (1997). A peer education model for teaching breast selfexamination to undergraduate college women. Cancer Nursing, 20(1), 49-63.

Meade, C.D., \& Calvo, A. (2001). Developing community-academic partnerships to enhance breast health among rural and Hispanic migrant and seasonal farmworker women. Oncology Nursing Forum, 28(10), 1577-1584.

Mitchell, G.J., \& Cody, W.K. (2002). Ambiguous opportunity: Toiling for truth of nursing art and science. Nursing Science Quarterly, 15(1), 71-79.

Mohr, W.K. (1995). Integrating esthetics into nursing: Literature as a suggested modality. Archives of Psychiatric Nursing, 9(6), 365372.

Oneha, M.F., Magnussen, L., \& Feletti, G. (1998). Ensuring quality nursing education in community-based settings. Nurse Educator, 23(1), 26-31.

Polit, D.F., \& Beck, C.T. (2003). Nursing research: Principles and methods (7th ed.). Philadelphia: Lippincott Williams \& Wilkins.

Thomas, B., Gold/Smith, S.B., Forrest, A., \& Marshall, R. (2002). Web site development: Applying aesthetics to promote breast health education and awareness. CIN: Computers, Informatics, Nursing, 20(5), 184-190.

Vezeau, T.M. (1994). Narrative in nursing practice and education. In P.L. Chinn \& J. Watson (Eds.), Art and aesthetics in nursing (pp. 163-188). New York: National League for Nursing Press.

Wood, R.Y., Duffy, M.E., Morris, S.J., \& Carnes, J.E. (2002). The effect of an educational intervention on promoting breast self-examination in older African American and Caucasian women. Oncology Nursing Forum, 29(7), 1081-1090.

Young-Mason, J. (2000). The art of healing and the healing power of art. Clinical Nurse Specialist, 14(4), 196-197. 